\title{
PROTEIN SYNTHESIS:
}

\section{Errors rectified in retrospect}

\author{
Kurt Fredrick and Michael Ibba \\ Department of Microbiology, the Ohio State Biochemistry Program and the Center for RNA Biology, \\ Ohio State University, Columbus, Ohio 43210-1292, USA
}

\begin{abstract}
During protein synthesis, mistakes in adding amino acids to the growing polypeptide chain are usually prevented. If they are not, a quality-control mechanism ensures premature termination of erroneous sequences.
\end{abstract}

For cells to flourish, the genetic code must be translated with great accuracy into the amino acids that proteins are made from. During translation, the cell's protein-synthesis factory the ribosome - carefully monitors the process by which new amino acids are added to a growing polypeptide chain. For each one, a specific trinucleotide (a codon) on messenger RNA is paired with a complementary anticodon on a transfer RNA, which at its other end carries the corresponding amino acid. Once codon-anticodon pairs have formed, the amino acid is chemically linked to the polypeptide chain by a peptide bond. At this point, it was thought that the quality-control duties of the ribosome were more or less complete. But Zaher and Green ${ }^{1}$ present evidence in this issue (page 161) that, even after peptide-bond formation, the ribosome can detect codon-anticodon mismatches and reacts by bringing the protein's synthesis to a premature end.

The matching of codons and anticodons by the ribosome is a tricky process, involving a certain amount of leeway (Watson-Crick wobble) to allow the reading of all 64 codons that make up the genetic code. So it is not surprising that, despite careful matchmaking, mistakes are sometimes made, resulting in misfolded or non-functional proteins that must be refolded or destroyed after translation is finished. During protein synthesis, mistakes are generally thought to occur at a rate of about 1 in every 20,000 amino acids, although levels can be higher or lower depending on the conditions ${ }^{2,3}$. Studies in different living systems support this estimated rate of error, whereas experiments with individual components of the protein-synthesis machinery in vitro have yielded less clear-cut results.

It was one such experiment that piqued Zaher and Green's interest. When looking at the formation of simple two-amino-acid peptides, they sometimes saw error rates as high as 1 in 2,000 - an order of magnitude higher than they had expected. To further explore these high error rates, they turned to an occasional mistake that is well documented in living systems: the erroneous translation of the AAU codon into the amino acid lysine, rather than aspara gine. They also began to look at the formation of longer peptides of up to four amino acids. Much to their surprise, they found that, once a mistake has been made, the ribosome becomes much less efficient at adding amino acids. So rather than continuing to grow, the nascent peptide chain was released from the translational machinery prematurely. 
Ribosomes contain three binding sites for their tRNA substrates: the aminoacyl (A) site, the peptidyl (P) site and the exit (E) site. During each round of amino-acid chain elongation, codonanticodon pairing allows entry of the correct tRNA into the A site (Fig. 1a). The nascent polypeptide chain bound to the tRNA at the P site is then transferred to the tRNA bearing a new amino acid at the A site, thereby lengthening the chain by one residue. This cycle of aminoacid addition is completed when the tRNA originally at the P site moves to the E site and the tRNA at the A site shifts to the P site, freeing up the A site for the next tRNA (Fig. 1a). The tRNA translocations are accompanied by mRNA movement by three nucleotides - or one codon - towards the E site. Iterative cycles of elongation occur until a stop codon, signalling the end, reaches the A site. Specific recognition of this codon by a primary release factor (known as RF proteins in the bacterium Escherichia coli) promotes hydrolysis of the now mature polypeptide from the P-site tRNA, a process called termination ${ }^{4}$.

In an effort to understand their puzzling observations, Zaher and Green ${ }^{1}$ studied several defined ribosomal complexes, made from purified components, in vitro. They find that complexes containing a mismatch between the anticodon and codon in the P site are susceptible to RF2mediated peptide release, despite the absence of a stop codon in the A site (Fig. 1b). Although slow, this reaction was stimulated considerably by the secondary release factor RF3, suggesting that it might be relevant in vivo, where both RFs are present.

Intriguingly, a sequence containing a mismatched codon-anticodon pair in the $\mathrm{P}$ site also stimulated further error - that is, incorporation of an amino acid despite the absence of correct codon-anticodon pairing ${ }^{1}$. Consequently, complexes containing codon-anticodon mismatches were made in both the $\mathrm{E}$ and $\mathrm{P}$ sites. Again, the authors observed high rates of RF-dependent peptide release in these complexes, suggesting that termination can efficiently compete with elongation. The net effect is that miscoding errors terminate translation prematurely, which is another means of quality control by the ribosome - retrospectively, following peptide-bond formation - to increase the fraction of functional proteins made.

How codon-anticodon mismatches in the P site (or P and E sites) stimulate further miscoding and peptide release remains unclear. Codon-anticodon pairing in these sites normally helps to maintain the correct reading of codons on mRNA. Mismatches could disrupt such systematic reading of mRNA, potentially allowing various codons to transiently occupy the A site as the mRNA slides through the ribosome unpaired. Another possibility is that mismatches generate a conformational signal in the ribosomal complex that alters the activities of the translation factors such as RF proteins. Indeed, earlier work ${ }^{5-7}$ showed that conformational changes in the ribosome regulate both the decoding of mRNA and its termination. Regardless of the precise mechanism involved, Zaher and Green's work ${ }^{1}$ reveals a facet of quality control in protein synthesis that depends on an unanticipated level of complexity in the workings of the ribosome.

\section{References}

1. Zaher HS, Green R. Nature 2009;457:161-166. [PubMed: 19092806]

2. Kurland CG. Annu. Rev. Genet 1992;26:29-50. [PubMed: 1482115]

3. Kramer EB, Farabaugh PJ. RNA 2007;13:87-96. [PubMed: 17095544]

4. Ogle JM, Ramakrishnan V. Annu. Rev. Biochem 2005;74:129-177. [PubMed: 15952884]

5. Youngman EM, He SL, Nikstad LJ, Green R. Mol. Cell 2007;28:533-543. [PubMed: 18042450]

6. Laurberg M, et al. Nature 2008;454:852-857. [PubMed: 18596689]

7. Weixlbaumer A, et al. Science 2008;322:953-956. [PubMed: 18988853] 


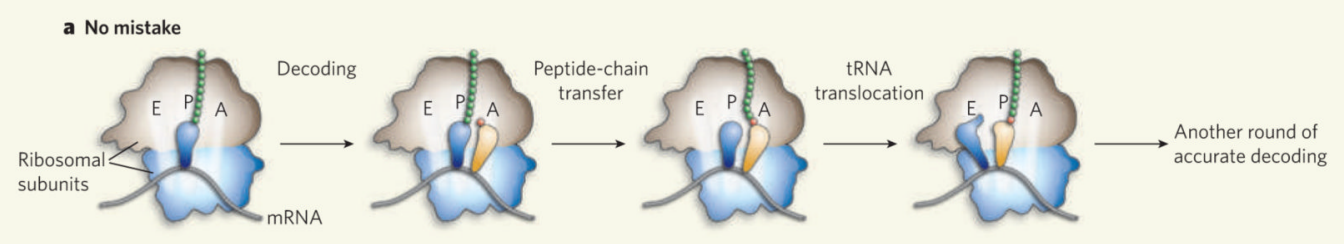

b Mistake

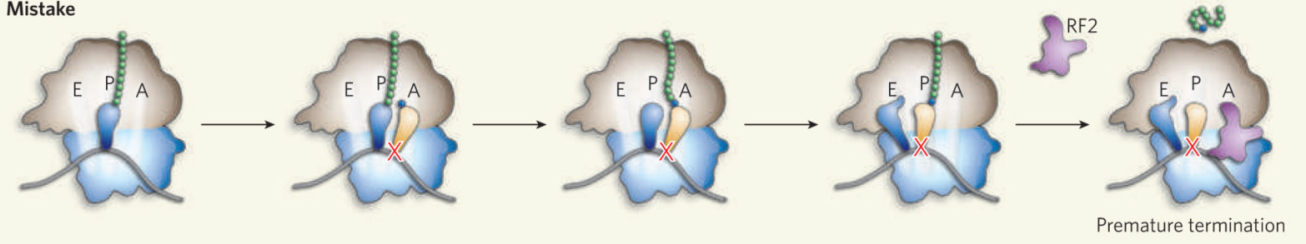

Figure 1. Ribosomal matchmaking

a, Normally, the correct tRNA (yellow) enters the A site of the ribosome and the appropriate amino acid (red) is incorporated into the growing peptide chain, which transfers from tRNA in the P site to the tRNA at the A site. Both tRNAs, as well as the mRNA, then shift towards the $\mathrm{E}$ site. $\mathbf{b}$, When mistakes are made and the mismatched codon-anticodon helix (indicated by a red cross) translocates to the $\mathrm{P}$ site, the ribosomal complex becomes susceptible to premature termination by translation factors such as RF2, and the erroneous sequence is prematurely released. 